\title{
Práctica científica en el Pacífico centroamericano. Los viajes de José Moraleda $(1802-1804)^{1}$
}

\author{
Macarena Ríos Llaneza²
}

\begin{abstract}
RESUMEN
Desde la perspectiva de la historia de la ciencia, este trabajo busca comprender la práctica científica del piloto Moraleda durante su comisión hidrográfica por el Pacífico centroamericano entre los años 1802 y 1804 . A partir del estudio de sus técnicas, instrumentos y conocimientos, se identifican las características del quehacer científico del navegante y del proceso de generación del conocimiento geográfico de América durante el siglo XVIII.
\end{abstract}

Palabras clave: José Moraleda, Pacífico centroamericano, navegación astronómica, práctica científica, siglo XVIII, siglo XIX.

\begin{abstract}
From the perspective of the history of science, this research seeks to understand the scientific field work carried out by captain Moraleda during his exploratory trip along the Pacific Ocean of Central America between 1802 and 1804. By means of studying his techniques, his instruments and his knowledge, we can identify the scientific characteristics proper of a marine's duty and those that yielded the geographic knowledge of America in the 18th century.
\end{abstract}

Key words: José Moraleda, Central America's Pacific Ocean, astronomic navigation, scientific field work, 18 th century, 19th century.

1 Agradecemos los comentarios, aportes y consejos del profesor Rafael Sagredo Baeza, bajo cuya dirección, y en el contexto del proyecto FONDECYT 1095221, preparamos este artículo, recibido el 17 de marzo de 2011, aceptado el 24 de junio de 2011 y corregido el 8 de agosto de 2011.
2 Instituto de Historia, Pontificia Universidad Católica de Chile (Chile). E-mail: mrios2@uc.cl 
Este artículo aborda los viajes de José Moraleda por el Pacífico centroamericano entre los años 1802 y 1804. A partir del estudio de su diario de viajes, identificaremos las características de la práctica científica ejercida por el piloto para el reconocimiento geográfico de América. De esta manera, pretendemos explicar su patrón de conducta, los métodos e instrumentos que utilizó y la información con la cual contaba para realizar su viaje. Así, analizaremos cómo fue el proceso de aplicación de las técnicas modernas de pilotaje, basadas en la Geodesia, la Astronomía y las Matemáticas, para el conocimiento científico de América.

Los viajes de José Moraleda por el Pacífico centroamericano se relacionan con una coyuntura histórica en la que el viaje, el poder y la ciencia se encuentran profundamente vinculados. A lo largo del siglo XVIII, la Corona española promovió, organizó y financió viajes y expediciones destinadas a conocer la realidad material de las colonias. A través de estos se pretendió obtener información acerca de los recursos existentes en las posesiones ultramarinas, cartografiar los territorios y mejorar la comunicación y el comercio con las colonias. De esta manera, los viajes Ilevados a cabo durante esta época cumplieron con un fin utilitario al vincular el conocimiento y sistematización de la realidad americana a la necesidad de ejercer un dominio efectivo de las posesiones ultramarinas (Sagredo y González, 2004).

Los protagonistas de estos desplazamientos fueron hombres formados en las técnicas modernas de navegación, producto de un proceso de institucionalización de la ciencia ilustrada a través de la marina española (Pimentel, 1998:81). José Moraleda, ejemplifica la nueva formación que se les dio a los navegantes desde mediados del siglo XVIII, producto de las reformas introducidas por Jorge Juan, que pretendían renovar los conocimientos náuticos y vincularlos con los astronómicos (Sellés, 1991).

Moraleda había nacido en 1747 en la provincia de Guipúzcoa y se cree que en el año 1760 ingresó a la Real Escuela de Navegación de Cádiz donde realizó sus estudios. En esta obtuvo la formación teórica en las técnicas científicas de navegación, a partir de la lectura de obras como El marinero instruido en el arte de navegar especulativo y práctico de Francisco Barreda, en la que se explica el uso de los instrumentos para llevar a cabo la navegación astronómica y los distintos métodos para obtener la latitud, longitud, variación de la aguja y los elementos necesarios para realizar el pilotaje. Desde 1764, José Moraleda comenzó a realizar distintos embarques a las Indias Orientales, recorriendo lugares como Achem, Malaca, Batavia, entre otros, y también a las Indias Occidentales, embarcándose a las costas de Veracruz, La Habana y Puerto Rico. La importancia de este tipo de travesías fue fundamental en la formación de los navegantes, puesto que constituían la instancia para la aplicación práctica de los principios teóricos aprendidos. Además, en estos embarques Moraleda adquirió la experiencia y el mérito que, años más tarde, le permitirían ascender al grado de piloto, encargándole comisiones de mayor importancia para la corona española (O'Donnell y Duque de la Estrada, 1990; Sagredo, 2008).

Fruto de sus travesías que se prolongaron hasta comienzos del siglo XIX, se conservan los diarios de viajes correspondientes a cada una de las comisiones (Cuadro $\mathrm{N}^{\circ} 1$ ). 
Cuadro $\mathrm{N}^{\circ} 1$

Comisiones de Moraleda entre los años 1772 y 1804

\begin{tabular}{|c|c|c|c|c|}
\hline $\begin{array}{l}\text { Nombre del diario de } \\
\text { viajes }\end{array}$ & $\begin{array}{l}\text { Duración del } \\
\text { viaje }\end{array}$ & Nombre de la nave & $\begin{array}{l}\text { Puerto de salida y } \\
\text { de llegada }\end{array}$ & Objetivos \\
\hline $\begin{array}{l}\text { Relación del derrotero } \\
\text { entre Cadiz y Lima, que } \\
\text { incluye una escala en } \\
\text { Concepción, además de } \\
\text { otras tavesías de Mora- } \\
\text { leda por el Mar del Sur } \\
\text { realizadas entre } 1777 \text { y } \\
1779\end{array}$ & 1772 a 1775 & $\begin{array}{l}\text { Nuestra Señora de } \\
\text { Montserrat }\end{array}$ & $\begin{array}{l}\text { Navegación por el } \\
\text { Mar del Sur }\end{array}$ & $\begin{array}{l}\text { Variados (transpor- } \\
\text { te de mercaderías } \\
\text { a Concepción, } \\
\text { conducción de } \\
\text { maderas para la } \\
\text { construcción de } \\
\text { naves desde Guaya- } \\
\text { quil al Callao entre } \\
\text { otros) }\end{array}$ \\
\hline $\begin{array}{l}\text { Diario de la navegación } \\
\text { desde el puerto de Callao } \\
\text { de Lima al de San Carlos } \\
\text { de la isla de Chiloé }\end{array}$ & 1787 a 1790 & $\begin{array}{l}\text { Desde Lima hasta } \\
\text { Chiloé en la nave } \\
\text { particular Nuestra } \\
\text { Señora de los Dolo- } \\
\text { res. En Chiloé inicia } \\
\text { los reconocimientos } \\
\text { a bordo de la falúa El } \\
\text { Socorro }\end{array}$ & $\begin{array}{l}\text { Desde puerto del } \\
\text { Callao al de San } \\
\text { Carlos, punto en } \\
\text { el cual inicia el } \\
\text { recorrido por el } \\
\text { archipiélago de } \\
\text { Chiloé }\end{array}$ & $\begin{array}{l}\text { Ayudar al gober- } \\
\text { nador Francisco } \\
\text { Hurtado en el } \\
\text { reconocimiento } \\
\text { del archipiélago } \\
\text { de Chiloé y en la } \\
\text { elaboración de una } \\
\text { cartografía de la } \\
\text { zona }\end{array}$ \\
\hline $\begin{array}{l}\text { Diario de la navegación } \\
\text { desde el puerto del } \\
\text { Callao de Lima al de San } \\
\text { Carlos de Chiloé, y de } \\
\text { este al reconocimiento } \\
\text { del archipiélago de Cho- } \\
\text { nos y costa occidental } \\
\text { patagónica comprendida } \\
\text { entre los } 41 \text { y } 46 \text { grados } \\
\text { de latitud meridional }\end{array}$ & 1792 a 1795 & $\begin{array}{l}\text { Desde el Callao al } \\
\text { puerto de San Carlos } \\
\text { en el paquebote } \\
\text { Santa Teresa. En San } \\
\text { Carlos inicia los } \\
\text { reconocimientos en } \\
\text { las piraguas Carmen } \\
\text { y Rosario }\end{array}$ & $\begin{array}{l}\text { Desde el Callao } \\
\text { al puerto de San } \\
\text { Carlos, y desde } \\
\text { este inicia el } \\
\text { reconocimiento de } \\
\text { la costa occidental } \\
\text { patagónica }\end{array}$ & $\begin{array}{l}\text { Reconocimiento } \\
\text { del archipiélago } \\
\text { de los Chonos y la } \\
\text { costa hacia el sur, } \\
\text { levantando planos } \\
\text { del territorio }\end{array}$ \\
\hline $\begin{array}{l}\text { Diario de viajes desde el } \\
\text { puerto del Callao a los } \\
\text { de Guayaquil y Panamá, } \\
\text { y de estos, al reconoci- } \\
\text { miento y demarcación } \\
\text { de las costas de Veragua, } \\
\text { Rica, Nicaragua y Gua- } \\
\text { temala }\end{array}$ & 1802 a 1804 & $\begin{array}{l}\text { A bordo de la corbe- } \\
\text { ta Castor. En Guaya- } \\
\text { quil se incorpora a } \\
\text { la comisión la goleta } \\
\text { Alavesa }\end{array}$ & $\begin{array}{l}\text { Desde el Callao } \\
\text { a Guayaquil, y } \\
\text { desde este hacia } \\
\text { el norte }\end{array}$ & $\begin{array}{l}\text { Reconocimiento } \\
\text { de las costas para } \\
\text { lograr una nave- } \\
\text { gación segura y } \\
\text { descripción política } \\
\text { de los lugares que } \\
\text { recorrió }\end{array}$ \\
\hline
\end{tabular}

Fuente: Elaboración propia.

\section{Los viajes de Moraleda por el Pacífico centroamericano}

Para comprender el interés de la corona española por el reconocimiento de la costa centroamericana es necesario aludir a la situación en la que se encontraban las colonias. En primer lugar, a fines del siglo
XVIII las Antillas y la zona septentrional del Pacífico constituían un "área de conflicto", debido al aumento de la presencia de holandeses, ingleses y franceses en la zona, que significaban una amenaza a los intereses del imperio español. Como reacción a este peligro, durante el gobierno de Carlos IV, se promovieron expediciones hidrográficas con 
el objetivo de cartografiar los territorios y obtener el conocimiento geográfico necesario para defender el imperio frente a la amenaza extranjera (Gutiérrez, 1991).

Siguiendo esta lógica, a Moraleda se le comisionó para levantar planos de las costas centroamericanas, que si bien habían sido cartografiadas en años anteriores por Alejandro Malaspina ${ }^{3}$, todavía existían ciertos puntos que no eran señalados en los planos antiguos ni modernos (Ugarte, 1802). Considerando las cartas elaboradas por el navegante italiano, Moraleda debía rectificar los resultados obtenidos por este y demarcar puntos intermedios a los obtenidos por Malaspina, de manera de lograr el "exacto padrón y reconocimiento de estas costas (Ugarte, 1802: capítulo 1, artículo 6). De esta manera, el reconocimiento geográfico de las costas centroamericanas constituía una forma de dominar y apropiarse de lo que constituía una vía esencial para la comunicación entre la metrópoli y las colonias: el mar (Figura $N^{\circ} 1$ ).

Por otra parte, además de esta estrategia defensiva vinculada a la cartografía de los territorios, la particularidad de los viajes realizados por Moraleda radica en que fue una comisión organizada desde las costas americanas, y por lo tanto, no solo vinculada a los intereses imperiales, sino también a la realidad de las posesiones ultramarinas. Es por esto que en las órdenes entregadas al piloto se señala que debía reconocer las costas desde el golfo de Panamá hasta Sonsonate con el objetivo de que "puedan exportarse más fácil y cómodamente los preciosos frutos del reino de Guatemala" (Ugarte, 1802, capítulo I, artículo 3$)^{4}$. De esta manera, el conocimiento del territorio aseguraría la posesión de las ru-

\footnotetext{
3 La expedición Malaspina se desarrolló entre los años 1789 y 1794. Como señala el mismo Malaspina y José de Bustamante, también miembro de la expedición, los objetivos de esta eran "la construcción de cartas hidrográficas para las regiones más remotas de América, y de derroteros que puedan guiar con acierto la poco experta navegación mercantil; y el otro la investigación del estado político de la América, así relativamente a España como a las naciones extranjeras" (Sagredo y González, 2004: 48)

4 Es importante recordar que la Audiencia de Guatemala experimentó un auge económico durante el siglo XVIII, gracias a la producción y comercio del cacao y del añil (Pinto, 1993).
}

tas comerciales, lo que no solo iría en beneficio de la corona española, sino que también aumentaría la actividad comercial entre las colonias americanas.

Además de los objetivos señalados, las instrucciones detallaban minuciosamente la forma en que el piloto debía realizar las mediciones, los instrumentos que debía utilizar y los cuidados necesarios en la aplicación de las distintas técnicas. Este punto resulta una particularidad con respecto a los viajes anteriores que había realizado Moraleda, en los que las instrucciones solo señalaban la derrota que debía seguir y las medidas de seguridad para garantizar los resultados. Las instrucciones para sus viajes por las costas centroamericanas, al definir la forma en que se debían realizar los reconocimientos, se transformaron en una guía de lo que se esperaba que fuera la práctica científica del navegante.

El detalle de las instrucciones también se explica por las características generales de la comisión. Esta estaba compuesta por 27 hombres divididos en dos naves, la Castor, a cargo de José Moraleda, quien además era comandante de la comisión, y la Alavesa, dirigida por Antonio Quartara. De esta manera, tal y como explica el Comandante del Apostadero del Callao de Lima, Tomás de Ugarte, las instrucciones estaban destinadas a uniformar los métodos de ambas naves. Homogeneizar la práctica científica ejercida en ambas corbetas, permitía la comparación de los resultados, procedimiento que resultaba fundamental para lograr la precisión de estos $^{5}$. Por otra parte, uniformar los métodos tenía como objetivo que durante la comisión se aplicaran los progresos en los conocimientos hidrográficos y las técnicas modernas de navegación como explicaremos a continuación (Ugarte, 1802).

\footnotetext{
5 Para profundizar en la importancia de la uniformidad en el método científico y su relación con la precisión de los resultados véase Valdeverde (2007).
} 
Figura $\mathrm{N}^{0} 1$

Mapa de Centroamérica que permite apreciar el ámbito en que se desenvolvió José Moraleda en su comisión hidrográfica desde 1802 a 1804

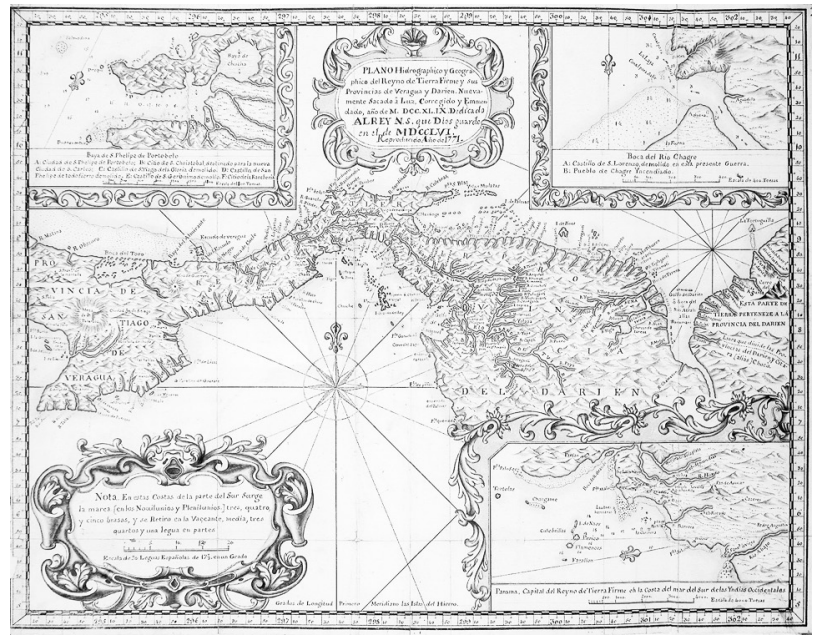

Fuente: Departamento de Cartografía y Artes Gráficas de la Real Academia de la Historia, España.

\section{Práctica científica del piloto: sus conocimientos y métodos}

La labor esencial del piloto durante el viaje consistía en trazar la ruta de la nave y determinar la posición de esta, o la desviación de la ruta en un instante dado. Para esto era necesario que el navegante solucionara los cuatro problemas que se le presentaban para conocer la situación del buque: latitud, longitud, rumbo y distancia (Sagredo, 2008). La resolución de estos problemas era fundamental, sobre todo en el caso de la navegación de altura, pues permitía que el piloto pudiera visualizar en las cartas la situación en la que se encontraba en el mar. Esta información permitía a los navegantes conocer la distancia a tierra y a los lugares a los cuales pretendía llegar, y así tener una idea del tiempo que demoraría en alcanzarlos.

En el caso de Moraleda, y como hemos señalado anteriormente, uno de los objetivos principales de su comisión era permitir una navegación más segura por la costa del Pacífico centroamericano, para lo cual era fundamental fijar la ruta que seguiría la nave. En su caso, el hecho de que existieran dos bajos, que además de constituir un peligro para la navegación, no estaban señalados en la cartografía de la época, incrementaban el interés por su trabajo.

Durante su viaje a Centroamérica, José Moraleda recalca que la ignorancia que tenían los navegantes acerca de estas zonas hacía que muchos prefirieran realizar una navegación bordeando la costa y, de esta manera, evitar que se perdieran en alta mar. El piloto señala que la mayoría de los navegantes solo se servían de un práctico de costa, prescindiendo de un piloto de altura, y que no tomaban en cuenta una dificultad propia del mar que navegaban, las corrientes. En su estadía en la ensenada de Damas, Moraleda recalca que la navegación costera podía servir en viajes desde el Callao a Pisco, Guayaquil y a algunos lugares como Santa Elena, sin embargo cuando emprendían viajes desde Guayaquil hasta Panamá o del puerto de Chocó a los de Veragua, estos tenían como resultado:

"Las desgracias que por lo común le son consecuentes (...) ya naufragando inesperadamente, ya haciendo una arribada 
ruinosa, o ya pereciendo algunos en el mar, faltos de subsistencia, por la prolongada navegación errante; pues aislados a solo el conocimiento de la tierra a corta distancia, y acaso a determinada configuración, las varias rápidas corrientes, que constantemente se experimentan en este golfo, o grande seno de la América Meridional, los alejan unas veces de la tierra, a distancias que no las conocen, y otras los apartan enteramente de su vista, dejándolos al arbitrio del impulso y dirección de las aguas" (Moraleda).

De esta manera, frente al peligro que significaban las corrientes y la falta de una cartografía más precisa de las costas que la comisión recorría, se hacía indispensable que el piloto contara con los instrumentos y conocimientos necesarios para llevar a cabo la navegación astronómica.

\section{Rumbo}

Este término definido como el "viaje, rastro, o línea, que forma la Nave sobre la superficie del Agua" (Barreda, 1786:71), había sido resuelto mediante el empleo de la brújula o aguja náutica.

Durante su viaje a Centroamérica, Moraleda utilizó una aguja conocida como de marcar. Esta era una caja cuadrada en cuyo centro estaba situaba la rosa de los vientos. En dos lados opuestos de dicha caja se ponían dos pínulas, que corresponden a dos tablillas. También, podía ser que en vez de estas la caja tuviera dos ventanas cuadradas. La utilidad de estas pínulas, o de las ventanas, era sostener dos hilos situados en forma perpendicular, dispuestos de tal manera que la sombra de uno de ellos, cuando se realizaba la marcación, pasara por el centro de la rosa y por lo tanto, del sol, dividiendo la rosa en dos (Barreda, 1786: 86).

Moraleda en su viaje determinó dos tipos de rumbo, el de su propia nave, y los rumbos o dirección de un determinado punto en la tierra o una nave con respecto a su embarcación. Además, siguiendo las instrucciones, cada vez que daba fondo en algún puerto, determinaba el rumbo de algunos puntos que se veían con respecto a su nave. Para realizar estas operaciones era necesario que el piloto tuviera conocimiento de las divisiones en el plano del horizonte, el cual se consideraba dividido en cuatro partes de noventa grados cada una, Ilamadas cuadrantes. Cada cuadrante a su vez estaba compuesto de ocho rumbos diferentes, con lo cual existían treinta y dos rumbos distintos. Por otra parte, era necesario el conocimiento de los vientos, que eran determinados por el piloto varias veces durante cada singladura. También se consideraba que existían treinta y dos tipos distintos, los cuales actuaban en sentido contrario al rumbo (Barreda, 1786: 71-82).

Al final de cada singladura, el piloto anotaba el rumbo directo que había seguido su nave, el cual debía estar corregido de la declinación magnética, entendida como la diferencia entre el norte geográfico y el norte que indicaba la aguja. Las instrucciones ordenaban a Moraleda que debía observar esta variación repetidas veces durante el día, comparar sus resultados con la corbeta Alavesa y luego obtener la correcta por medio de un promedio (Ugarte, 1802: capítulo 2, artículo 23). Este mismo patrón se repite en las mediciones de variación que realizó en los reconocimientos de determinados lugares, entre ellos en el fondeadero de la bahía de Panamá, en el cual a partir de varias observaciones obtuvo un promedio que dio como resultado aproximado $9^{\circ}$ de variación $\mathrm{NE}$ (Moraleda).

En las singladuras en que el piloto hace referencia a la obtención de la variación, especificaba que esta había sido observada al ponerse el Sol, momento en el cual medía la amplitud del astro, es decir, el ángulo formado por el meridiano que se utilizaba como base y el plano vertical que cruzaba la observación del navegante al Sol. Cuando se medía la amplitud también debía observarse el punto cardinal en la aguja, lo que más tarde determinaría si los resultados debían restarse o sumarse (Barreda, 1786. 112:113). Además, para corregir el rumbo también era necesario medir las declinaciones del Sol, obtenidas por medio de la observación de la latitud (Barreda, 1786: 90). Luego, para saber la declinación exacta el piloto calculaba la diferencia entre la obtenida por observación y la obtenida por estima. 
Las veces que Moraleda hace referencia a la observación de la variación se puede ver que coincide que todas las variaciones son al noreste, con lo cual se puede deducir que se le presentaron los siguientes casos o problemas propios de una travesía ${ }^{6}$ :

1. Amplitud occidua al norte y la marcación de la mañana al norte o la de la tarde al sur, se suman los grados de la amplitud y la marcación, y el resultado correspondía a la variación.

2. Amplitud occidua al sur y marcación de la mañana al norte o marcación de la tarde al sur, se resta la amplitud a la marcación.

3. Amplitud occidua al norte y la marcación de la mañana al sur o la de la tarde al norte, se restan los grados de la amplitud y de la marcación.

4. No midió amplitud occidua y la variación era igual a los grados que obtuvo por la marcación en la mañana al norte o en la tarde al sur.

5. No vio tierra con lo cual no realizó marcaciones y la variación es igual a los grados que obtuvo por la medición de la amplitud occidua. Este caso creemos que se le presentó varias veces a lo largo del viaje, pues en su diario se ve que el piloto realizó durante algunos días navegación de altura sin ver tierra, aunque igualmente da a conocer su variación.

También para realizar la corrección debía considerarse la declinación del día anterior o siguiente. Es por esto que en algunas singladuras se puede ver la siguiente situación en que el rumbo es corregido un día después:

"El rumbo directo de la singladura fue $\mathrm{S}$ $41^{\circ} 45^{\prime} \mathrm{O}$, distancia 38 3/4 millas, observé la latitud de $2^{\circ} 14^{\prime} 00^{\prime \prime}$, y porque difiere de la estima 12'55" al Norte, corregimos con el meridiano, y resulta rumbo corregido en ésta

\footnotetext{
6 Para la explicación de estos casos seguimos a Barreda, 1786: 113-114.

(E.g. Moraleda 1802-1804: singladura del 17 a 18 de febrero de 1803).
}

y la anterior singladura $S 45^{\circ} 50^{\prime} \mathrm{O}^{\prime \prime}$ (Morale$d a, s / f)$.

La lectura de sus diarios de viajes, nos permite concluir que Moraleda solo corrigió el rumbo cuando la diferencia entre la latitud observada y la latitud obtenida por estimar era mayor a cuatro minutos.

Creemos que es necesario recalcar que a diferencia de su diario de viaje a Lima, Moraleda en sus viajes a Chiloé, la Patagonia y a las colonias centroamericanas no fue anotando en una tabla a cada hora los vientos y los rumbos, sino que establecía estos términos de manera más general. En las noches, por ejemplo, ya no detallaba su situación en cada momento sino que la daba a conocer en términos más amplios. Tal vez, porque ya tenía la experiencia que le permitía prescindir de llevar sus anotaciones tan detalladamente como la tabla requería.

\section{Distancia}

Para resolver este término José Moraleda contaba con una corredera, que consistía en un trozo de madera unido a una cuerda que estaba dividida en porciones iguales por medio de nudos, cuyas medidas correspondían a la vara de Castilla ${ }^{7}$. Para el uso de este instrumento debía tirarse la cuerda al mar e irse contando los nudos de acuerdo a un intervalo de tiempo que era medido por una ampolleta o reloj de arena (Sélles, 2000: 47).

Las instrucciones ordenaban al piloto realizar este procedimiento cada media hora y con más frecuencia en los casos que fuera necesario. Además, las distancias obtenidas y las medidas de la corredera entre ambas naves debían ser comparadas por lo menos una vez a la semana y cada vez que dieran a la vela desde algún surgidero. Lo mismo sucedía con las ampolletas que debían rectificarse de acuerdo a la marcha de los relojes (Ugarte, 1802: capítulo 2, artículo 22).

Si bien Moraleda no especifica en su diario el momento en el que se realizan estas mediciones, al final de cada singladura da a

7 Moraleda: advertencias, $\mathrm{N}^{\circ} 6$. 
conocer la distancia recorrida y la distancia de la nave con respecto a algunos puntos en Tierra. Además, luego de determinar la dirección de las corrientes, da a conocer el abatimiento, es decir, el desvío de la nave con respecto al rumbo que seguía, el cual era producido por el efecto de las corrientes. Estas en algunos casos produjeron modificaciones importantes en el cálculo de la distancia y el rumbo de la nave:

"Demora la Punta San Francisco Solano al $\mathrm{N} 25^{\circ} \mathrm{O}$, distancia de 14 leguas, y distamos de la tierra más inmediata 5, esta, ni otra alguna se ve estando el tiempo sumamente claro, luego nuestra distancia a ella, es mucho mayor, a efecto probable de la corriente que creemos nos ha conducido algunas millas muy próximamente al Oeste en la singladura" (Moraleda).

A pesar de algunas singladuras en las que se presentó el problema de abatimiento, la medición de la distancia no presentó una mayor dificultad durante el viaje, a excepción de la salida de Panamá en la que el piloto detalla:

"No pudo observarse la latitud, y fue la llegada de estima 7०30'52", y la longitud $73^{\circ} 39^{\prime} 10^{\prime \prime}$, pero resultando las últimas demarcaciones la latitud fue de $7^{\circ} 16^{\prime} 45^{\prime \prime}$, y la longitud de $73^{\circ} 41^{\prime} 30^{\prime \prime}$, se deduce habernos conducido la corriente al $\mathrm{S} 9^{\circ} \mathrm{O}$, la distancia de 14 1/3 millas, bien que parte de ésta, la concibo acción del viento y mal medida de la corredera por la desigualdad de aquel" (Moraleda, s/f).

El hecho de que la dirección de las corrientes haya sido señalada en casi todas las singladuras permite deducir que el piloto cumplió con la instrucción de medir la distancia varias veces durante el día, pues en su viaje desde Cádiz al Callao, Moraleda señalaba que sin esta constancia no era posible determinar la verdadera dirección de las corrientes $^{8}$. Además, él mismo señalaba que

8 E.g. Moraleda (1772-1779), Viaje al Puerto del CaIlao de Lima, en la Urca (barca) afragatada del rey, del porte de 40 cañones, nombrada Nuestra Señora de Montserrat año de 1772. Salida desde el río el ser sistemático en esta medición permitía obtener un resultado correcto pues,

"Cuanto más tiempo media entre una y otra, más se imposibilita el aproximarse a la verdadera distancia navegada, y por consiguiente no puede calcularse esta por aproximación a la que realmente anda el buque" (Moraleda: día 13 a 14 de noviembre de 1777).

\section{Latitud}

Este término corresponde a la distancia existente entre la línea equinoccial y un punto determinado del planeta (Barreda, 1786: 158). Su obtención fue resuelta de manera más exacta a mediados del siglo XV mediante la observación de la altura de los astros, como la estrella Polar, desde distintos lugares, y a partir de la diferencia entre los resultados se obtenía la distancia en leguas existente entre éstos (Sélles, 1994: 43).

José Moraleda, en el viaje que nos sirve de fuente, llevó distintos instrumentos de observación para realizar las mediciones de este término. En primer lugar, las instrucciones señalan que el navegante contaba con octantes (Ugarte, 1802: capítulo 2, artículo 62), que consistían en un arco de cuarenta y cinco grados, los que a su vez estaban divididos en noventa partes iguales y estas subdivididas en minutos. Al centro del arco tenía una regla, llamada alidada, cuyo movimiento señalaba las distintas medidas, obtenidas a partir de la observación por medio de un sistema de espejos móviles y fijos dispuestos en forma perpendicular a la alidada. Por último, contaba con dos pínulas, utilizadas para dirigir la vista dependiendo si la observación era realizada de espaldas o de frente al astro (Barreda, 1786: 169-171).

También el piloto llevaba consigo un sextante, cuya diferencia con el anterior consistía en que se encontraba dividido en sesenta grados. Además, contaba con un horizonte artificial, complemento para los instrumentos de reflexión, que correspondía a un recipien-

Guayaquil hacia el puerto del Callao, día 5 a 6 de noviembre de 1777 . 
te que contenía mercurio, y que por medio de un plano horizontal, corregía los efectos de la irregularidad del horizonte cuando se tomaban las alturas de los astros con respecto a este (Dirección General del Instituto Geográfico Nacional, 2002: 56 y 117).

Para realizar las observaciones por medio del sextante se buscaba, a través del espejo fijo la medianía del horizonte. Con el otro espejo se observaba un astro determinado, mientras se movía la alidada hasta el punto en que se hacía coincidir el astro con la mitad del horizonte que se observada por el otro espejo. Los grados que marcaba la alidada correspondían a la elevación del astro sobre el horizonte. Si la observación se realizaba de espaldas, la diferencia radicaba en que el horizonte se observaba por medio del espejo móvil y por el fijo el astro (Cf. Barreda, 1786: 173-176).

A Moraleda en su viaje se le encargó observar la latitud todos los días mediante la medición de la altura del Sol al mediodía (Ugarte, 1802: capítulo 2, artículo 40). Por la forma en que obtiene la variación magnética, se puede deducir que también el piloto realizaba este método a la puesta de Sol. Estos cálculos debían corregirse de la refracción, entendida como la desviación de los rayos de luz cuando pasaban de un medio a otro. La refracción se restaba al resultado de la altura del Sol (Cf. Barreda, 1786: 184) y de esta manera se obtenía un resultado que Moraleda señalaba al final de cada singladura con el término "latitud observada". En algunos casos, la observación de la latitud se realizaba por más de un observador, como ocurrió con la medición en la isla Gorgona (Moraleda, día 21 a 22 de mayo de 1803, nota).

Además, tal y como se lo ordenaban las instrucciones, en las noches despejadas debía realizar observaciones de la altura meridiana de otros astros. Esto es precisamente lo que realizó el piloto en su travesía desde el río Guayaquil a la bahía de Panamá, ocasión en que el buen tiempo le permitió obtener la latitud por medio de la observación de la estrella Sirio (Moraleda, día 3 a 4 de marzo de 1803). La medición fue realizada dos días seguidos por tres observadores al mismo tiempo, lo que facilitaba la división de las tareas para tomar horarios, medir la altura de la estrella sobre el horizonte y calcular la distancia angular de esta con respecto a otro astro tomado como referencia. Además, el beneficio que tenía la elección de dicha estrella es que es brillante y la más cercana a la Tierra, lo que reducía el margen de error en los resultados. Estas dos singladuras son prácticamente las únicas en las que el piloto señaló la hora exacta en la cual realizó la medición, el primer día a las 19:48 horas y el segundo a las 19:04 horas.

Moraleda también obtenía la latitud por medio de la estima, para lo cual era necesario conocer el resultado final de la latitud del día anterior y las demarcaciones realizadas durante el día, datos que a su vez permitían deducir las distancias navegadas y por lo tanto, la diferencia de latitud obtenida el día anterior (que es comúnmente la observada) y el día de la estima. El cálculo de la estima era un trabajo que requería mucho cuidado y que incluso podía prolongarse durante días. Podía ocurrir, como se dijo anteriormente, que la latitud observada no coincidiera con la de estima, con lo cual se realizaba una corrección en base a un promedio entre los dos resultados conocido como latitud media (Ciscar, 1811: 99). En la mayoría de las singladuras el piloto pudo calcular la latitud por observación, sin embargo hay algunas excepciones en que el estado de la atmósfera se lo impidió con lo cual el resultado final se basaba en la latitud de estima.

Este término también podía calcularse por medio de las demarcaciones realizadas a puntos en la tierra, como explicaremos en las páginas siguientes.

\section{Longitud}

Este término se refiere a la distancia angular existente entre el Primer Meridiano y el lugar donde se encuentra la nave o un punto en la tierra (Barreda, 1786:195). El establecimiento de este Meridiano base se había transformado en un problema político, técnico-industrial y científico, en el que los países europeos rivalizaban, lo que dificultaba la unificación de los resultados (Martínez-Cañavate, 1990: 32). En sus viajes a Centroamérica, Moraleda utilizó como Primer Meridiano el del Observatorio Real de la 
Marina de Cádiz, al igual que en su comisión a la Patagonia occidental.

Según lo señalado por el piloto, él obtuvo la longitud a partir de distintos métodos. El primero de ellos fue la observación de las distancias lunares, técnica por la cual realizaba tres observaciones al mismo tiempo: medía la distancia angular de la Luna a un astro determinado, la altura de la Luna y la del astro sobre el horizonte. El problema de este método era que los resultados de la distancia se veían entorpecidos por los efectos de la refracción y paralaje. Para corregirlo, se realizaba una operación trigonométrica basada en la obtención del coseno de la distancia verdadera a partir del seno y coseno de noventa grados menos la altura del astro, y el seno y coseno de noventa grados menos la altura de la Luna (Sellés, 2000: 177-179). En algunos casos vemos que la obtención de la longitud por observación requería un cálculo que podía demorarse desde horas hasta un día 9 .

Otro método utilizado por el piloto español es la medición de tres alturas del Sol, dos veces cada día, una en la mañana y otra en la tarde, bajo la condición que las horas elegidas tuvieran la misma diferencia con respecto al mediodía. Por esto, Moraleda realizaba las mediciones a las 6:00 hrs y a las 18:00 hrs y el promedio de los horarios entre las alturas obtenidas durante la mañana y durante la tarde correspondía a la longitud de la nave al mediodía (Ciscar, 1811: 119).

Como se ve, el factor principal de este método era la medición del tiempo. Para esto Moraleda contaba con dos relojes que le permitieron vincular por medio del tiempo distintos puntos en la Tierra ${ }^{10}$. El embarcado en La Alavesa correspondía a el $N^{\circ} 262$ de Arnold. Desde el principio Moraleda no tuvo confianza en la máquina, pues antes de iniciar el viaje señala que

"Acaba de salir de las manos de un relojero, no ha habido tiempo de observarla,

9 E.g. Moraleda (s/f), en la cual Moraleda pide la longitud observada a la goleta La Alavesa y esta había terminado el cálculo del día anterior, mientras que en el de esa singladura demoró dos horas y media.

10 Para una obra que hace accesible el problema de la medición de la longitud, véase Sobel (1998). a consecuencia es muy probable su defectuosa marcha, y consiguiente inutilidad" (Moraleda, advertencias, № 1).

Tal y como lo previno el navegante, dicha máquina no favoreció al desempeño de la comisión, pues no se pudo arreglar su marcha.

El reloj embarcado en la Castor correspondía a el $n^{\circ} 154$ de Arnold acerca del cual el piloto señala que

"Ha cedido al mejor desempeño del servicio, por la bondad, que asegura observada en dicha máquina, en el espacio de seis años" (Moraleda: advertencias, $\mathrm{N}^{\circ} 1$ ).

A pesar de la confianza del navegante en este reloj, durante las primeras singladuras no pudo utilizarse porque no se había corregido su marcha. Sin embargo, esto no representó una dificultad ya que para los cálculos de esos días utilizó el reloj de Alexander von Humboldt, a quien el piloto tuvo que transportar en su viaje desde Callao a Guayaquil con destino a México ${ }^{11}$.

11 Con respecto a la relación entre Humboldt y Moraleda, cabe señalar, que el piloto se refiere a él al inicio de su diario de viajes, señalando que será pasajero de la nave hasta su Ilegada a Guayaquil, desde donde el prusiano se dirigiría a Acapulco. Luego de hacer una breve biografía de Humboldt, Moraleda señala que el objetivo de los viajes del prusiano consistía en "dar una física general del mundo" mientras Bonpland "observa y trata la parte de Historia Natural, y de costumbres de los diversos países, que discurren, y la Medicina". Luego el piloto agrega que para lograr estos objetivos era necesario "más detención que la que emplean en los viajes que hacen al intento, pues en ellos han corrido, si con intrepidez, también como un torrente rápido, una inmensidad de país en muy poco tiempo". Posteriormente, Moraleda manifiesta cierta desconfianza a las mediciones de la temperatura marítima que Humboldt había realizado en el Callao diciendo que "muchas razones podrá hallar el Barón, para esa particular frialdad; pero me parece que una atmósfera, casi constantemente toldada como esta, debe entrar en el número de las demás". Los juicios que emitió Moraleda acerca de la forma de proceder de Humboldt resultan interesantes, pues reflejan que para el piloto, el tiempo era un factor esencial para lograr la precisión en el quehacer científico. Esta opinión, junto a las referencias del reloj del viajero prusiano y a las comparaciones con sus resultados, constituyen las únicas alusiones del piloto con respecto al pasajero. 
Durante los primeros días de viaje, el reloj de Humboldt fue utilizado en cada singladura. Su importancia y exactitud queda manifestada en que en todos los casos el piloto prefirió ese resultado a los que obtuvo él mismo por sus instrumentos o por sus operaciones matemáticas:

"Por el reloj del Barón Humboldt se ha deducido la longitud de $74^{\circ} 53^{\prime}$, que respecto a la vista y rumbo a que esta la tierra, y la situación que dan a esta, nuestras citadas cartas del Depósito Hidrográfico, es bastante exacta la longitud observada dicha, y manifiesta que el error de la estima, es próximamente de 8 leguas, el buque al Occidente de ella" (Moraleda: día 28 a 29 de diciembre de 1802).

Asimismo, en algunas singladuras es posible apreciar que, a pesar de que Moraleda entrega una longitud obtenida por sus propios métodos, igual detalla la obtenida por medio del reloj del viajero prusiano:

"Por las demarcaciones del mediodía, se deduce la latitud de $4^{\circ} 35^{\prime} 30^{\prime \prime}$, y la longitud de $75^{\circ} 3^{\prime} 00^{\prime \prime}$ (...) y que las longitudes que se han deducido por el reloj del Barón Humboldt, con la despreciable diferencia de 2 ' equinocciales, han sido las mismas que señalan nuestras citadas cartas del Depósito Hidrográfico" (Moraleda: día 29 y 30 de diciembre de 1802).

Lo anterior da cuenta de la confianza que José Moraleda tenía en los instrumentos y en las mediciones realizadas junto a Humboldt, pues no solo las incluyó, sino que también se transformaron en un parámetro de comparación con sus propios resultados y con las cartas del Depósito Hidrográfico ${ }^{12}$, que

\footnotetext{
12 Esta institución había sido creada en España el año 1797 con el objetivo de renovar y perfeccionar la cartografía náutica, publicar las más aptas para la navegación y de estimular la preparación de nuevos derroteros, que favorecerían la lectura de las cartas náuticas. Su creación respondía a una política estratégica de proteger los intereses imperiales a través del desarrollo naval. A esta institución estuvieron vinculados destacados hombres de la Marina, tales como Alejandro Malaspina y Jorge Juan, quienes contribuyeron al desarrollo de las actividades del Depósito (Gutiérrez, 1991; Rivera y Martín-Merás, 1992: 213-224).
}

formaban la base de datos de su comisión. La confianza que el piloto español depositó en los resultados obtenidos por Humboldt adquiere aun más importancia si se considera la rigurosidad con que Moraleda realizaba los distintos cálculos y lo estricto que resultaba a la hora de detallar sus resultados.

Creemos que en su primera salida desde Guayaquil a Panamá, la marcha del reloj $\mathrm{N}^{\circ}$ 154 ya había sido arreglada, pues en la singladura del 21 a 22 de febrero de 1803, el piloto alude a los resultados obtenidos por dicho reloj, los cuales fueron comparados con las cartas, obteniendo pequeñas diferencias.

El cuidado de los relojes requería de muchas precauciones, ya que su movimiento se podía ver alterado por el cambio de temperaturas y por el movimiento de la nave. Para prevenir esta situación, Moraleda debía guardar el reloj bajo llave en su camarote y sacarlo todos los días a las 10:00 hrs para darle cuerda. Además, debía verificar su marcha de acuerdo a las observaciones de alturas, las que según lo señalado en las instrucciones, debían tomarse antes de las 8:00 hrs. Esta comparación permitía al navegante examinar si existía alguna diferencia que delatara algún atraso o adelanto del instrumento.

Otro aspecto importante en la medición de la longitud, era que el resultado obtenido debía ser comparado entre ambas naves, de esta manera podemos ver que la práctica científica no es ejercida solamente por una persona, sino que es una actividad que involucra a un grupo, lo que permitía reducir los márgenes de error:

"Se le hizo señal para que virase (...) y se le pidió la longitud por la correspondiente señal; a las 1 1 1/2 pareció haber señalado $71^{\circ} 49^{\prime}$, pero impidiendo el reflejo del sol discernir precisamente los colores de las banderas, cargamos las mayores y facheamos la sobremesana para aguardarla; a poco más de las dos pasó por la popa y expresó a la voz la longitud citada" (Moraleda: 27 a 28 de enero de 1804).

Además de obtener la longitud por medio de la observación, el piloto la obtenía por medio de la estima. Para esto, Moraleda requería conocer la longitud final del día 
anterior que junto a la distancia recorrida, la latitud y el rumbo, le permitían deducir cuál debía ser la longitud de la nave. El cálculo de la longitud de estima se hacía más necesario si se considera que algunas veces la intensidad de las corrientes y lo nebuloso del tiempo impedían al piloto observar la longitud:

"Aún al buen reloj y al hábil observador, deja inválidos el estado frecuente de la atmósfera en todo este golfo o grande ensenada, no permitiendo tomar en muchos días, las alturas y distancias debidas para deducir la longitud verdadera; y por consiguiente la dirección y acción de la corriente y vecindad a los riesgos en que ellas ponen" (Moraleda: día 4 a 5 de marzo de 1803).

El objetivo de medir la longitud por medio de la observación y de la estima, era que la comparación entre ambos resultados permitía conocer el apartamiento de meridiano, es decir, la medida del arco comprendido entre dos meridianos, resultado que a su vez permitía obtener la distancia recorrida, la dirección y la velocidad de las corrientes (Cf. Ciscar, 1811: 100). Considerando la intensidad de las corrientes de la costa centroamericana que recorrió Moraleda, la corrección de la longitud de estima (así como de la latitud de estima) era fundamental para no perder la orientación en el mar y sufrir un naufragio como le había sucedido a la fragata Santa Leocadia la que, a juicio del navegante, había naufragado por el desconocimiento e ignorancia de los pilotos de esos mares acerca de la dirección de las corrientes (Moraleda, s/f: día 11 a 12 de febrero de 1803). Como expresa él mismo la variedad de las direcciones de las corrientes

"Ha retraído siempre, de hacer correcciones a la estima, suponiendo al arbitrio conjetural el curso de las aguas, no obstante la ley general que les imponen los vientos periódicos y la dirección de las costas" ((Moraleda, s/f: día 26 a 27 de diciembre de 1802).

A pesar de la rigurosidad con la que Moraleda llevaba los cálculos, algunas veces las corrientes provocaban que la longitud de estima y la observada, o la obtenida por demarcaciones, tuvieran diferencias considerables que producían que el piloto llegara antes a tierra con su estima que en la realidad:
"Esta longitud nos presenta ya sobre la tierra, no se ve ni aún el menor indicio de ella, es, pues positivo que las corrientes experimentadas en esta y las dos anteriores singladuras, han girado a rumbos del tercer cuadrante, y siendo probable, por la experiencia de los pilotos más juiciosos de estos mares, y algo de la propia, que la dirección de ellas es próxima al SO" (Moraleda: día 1 a 2 de febrero de 1804).

Cuando las diferencias entre la longitud observada y la de estima eran aproximadamente un minuto, el piloto señalaba que la corriente no había alterado la dirección de la nave:

"Resulta la longitud de $74^{\circ} 39^{\prime} 18^{\prime \prime}$, por las demarcaciones últimas es la latitud 29' Sur, y la longitud $74^{\circ} 40^{\prime} 30^{\prime \prime}$, lo que nos manifiesta así, no haber impulso de corriente alguna en 24 horas" (Moraleda: día 12 a 13 de febrero de 1803).

La lectura de su diario de viajes nos permite establecer que las correcciones eran realizadas cuando las diferencias entre las longitudes obtenidas eran superiores a un minuto treinta segundos, aunque hay algunas excepciones en las que si bien la diferencia de minutos era amplia, no se realizó la corrección en la misma singladura, sino que el cálculo era corregido en días posteriores (E.g. Moraleda, singladura del 9 a 10 de marzo de 1803). Los resultados obtenidos de las mediciones de la latitud también influían en el cálculo del desplazamiento por corriente, es por esto que en algunas singladuras Moraleda señala la distancia y rumbo del desplazamiento a pesar de que los resultados del cálculo de la longitud eran similares (Moraleda, s/f: día 17 a 18 de mayo de 1803).

A continuación explicaremos el método que utilizaba el piloto para corregir el desplazamiento por las corrientes a partir del ejemplo de la singladura del día 20 a 21 de junio de 1803:

"El rumbo directo de la singladura fue S $8^{\circ} 40^{\prime} \mathrm{O}$, distancia 17 3/4 millas, no pudo observase la latitud, y resultó ser la llegada de estima $2^{\circ} 23^{\prime} 6^{\prime \prime}$, y la longitud $74^{\circ} 38^{\prime} 56^{\prime \prime}$, pero siendo esta $74^{\circ} 50^{\prime} 00^{\prime \prime}$, y la latitud $2^{\circ} 13^{\prime} 00^{\prime \prime}$, por la demarcación del mediodía se deduce habernos conducido la corriente al $\mathrm{N} 49^{\circ} \mathrm{O}$, la distancia de 14 2/3 millas en las 24 horas precedentes" (Moraleda, singladura día 21 a 22 de junio de 1803). 
Figura $\mathrm{N}^{\circ} 2$

Método utilizado por Moraleda para corregir el desplazamiento por las corrientes marinas

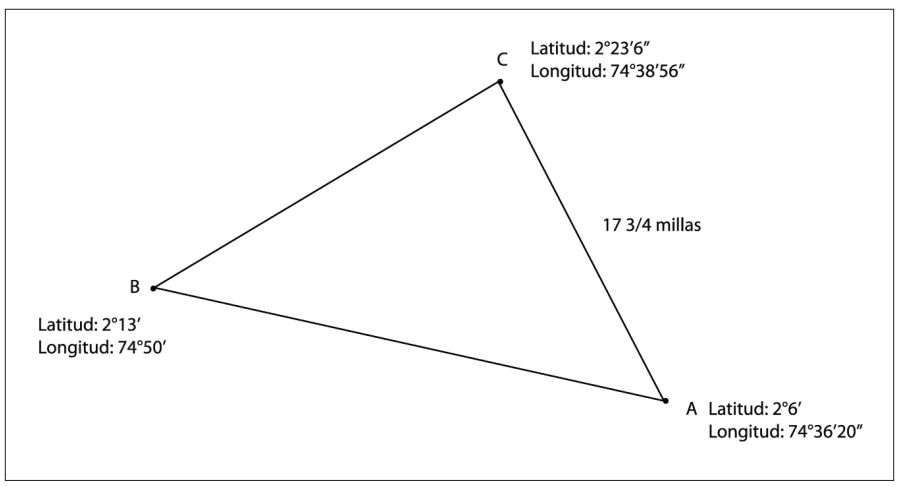

Fuente: Elaboración propia

En el triángulo de la Figura $N^{\circ}$ 2, el punto A corresponde a la situación de la nave en la singladura anterior, con respecto a la cual el piloto pudo obtener la situación de la nave de aquel día, el punto B a la situación de la nave de acuerdo a las demarcaciones realizadas ese día, y el punto C a la situación de la nave de acuerdo a sus cálculos de longitud y latitud. Las reglas del pilotaje establecían que el conocimiento de dos términos (latitud, longitud, rumbo y distancia) permitía deducir los otros dos. En este caso, Moraleda contaba con la latitud y la longitud. Como la navegación se efectuaba en el segundo cuadrante, por la diferencia de latitud entre el punto B y el C, el piloto conocía que su latitud había aumentado $10^{\prime} 6^{\prime \prime}$, con lo cual la corriente lo había desplazado al norte. Asimismo, la diferencia de longitud entre dichos puntos, le permitían establecer que su longitud había disminuido en $11^{\prime} 44^{\prime \prime}$, lo que lo habían hecho avanzar al Oeste. De esta manera, Moraleda podía conocer la dirección al NO que había provocado el desplazamiento por corriente.

Luego, las medidas de latitud y longitud debían trasladarse a minutos y estos a millas, considerando que en la época una milla náutica correspondía a un minuto (Cf. Sagredo, 2008). En la Figura $N^{\circ} 3$, se muestra las medidas en millas, considerando que la variable " $\mathrm{x}$ " corresponde a la longitud y la variable " $y$ " a la latitud:

Figura $\mathrm{N}^{\circ} 3$

Transformación de la latitud y longitud a millas naúticas

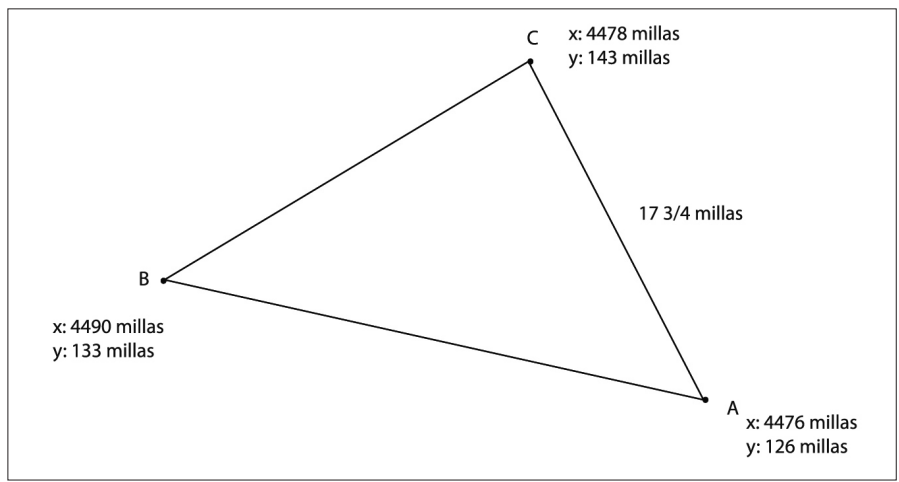

Fuente: Elaboración propia. 
Posteriormente, se podían obtener las distancias verticales, horizontales y ortogonales entre los distintos puntos, a partir de la dife- rencia entre las variables de " $x$ " e " $y$ " entre ellas (Figura $\mathrm{N}^{\circ} 4$ ).

Figura $\mathrm{N}^{\circ} 4$

Obtención de distancias verticales, horizontales y ortogonales

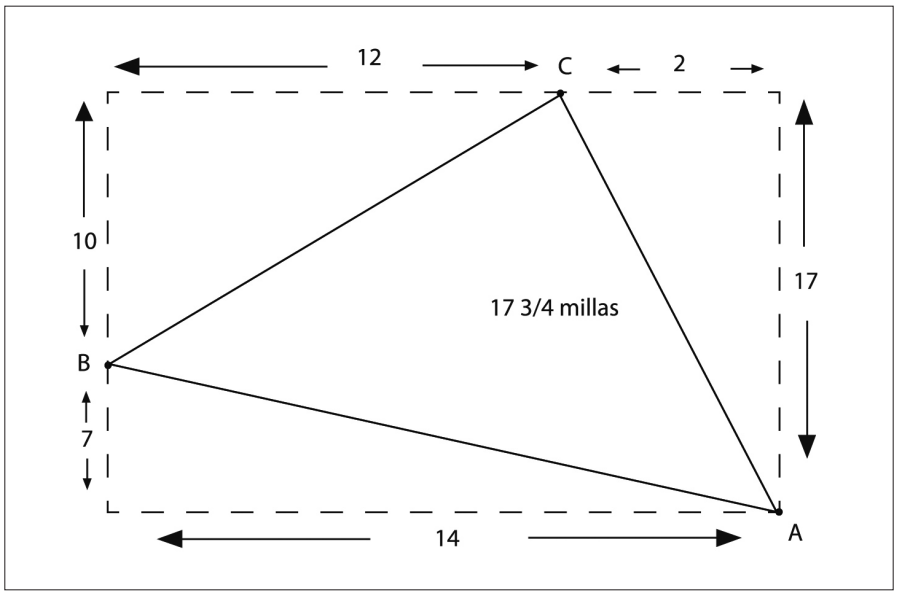

Fuente: Elaboración propia.

Obtenidas las distancias se podían calcular los valores que permitirían obtener los ángulos interiores del triángulo ABC, entre ellos el ángulo del rumbo del desplazamiento. La operación que se requería para esto era cal- cular la arcotangente. Esta consiste en una relación entre el ángulo sustentado por un lado opuesto y uno adyacente en todo triangulo rectángulo (Figura $N^{\circ} 5$ ).

Figura $\mathrm{N}^{0} 5$

Cálculos de la arcotangente

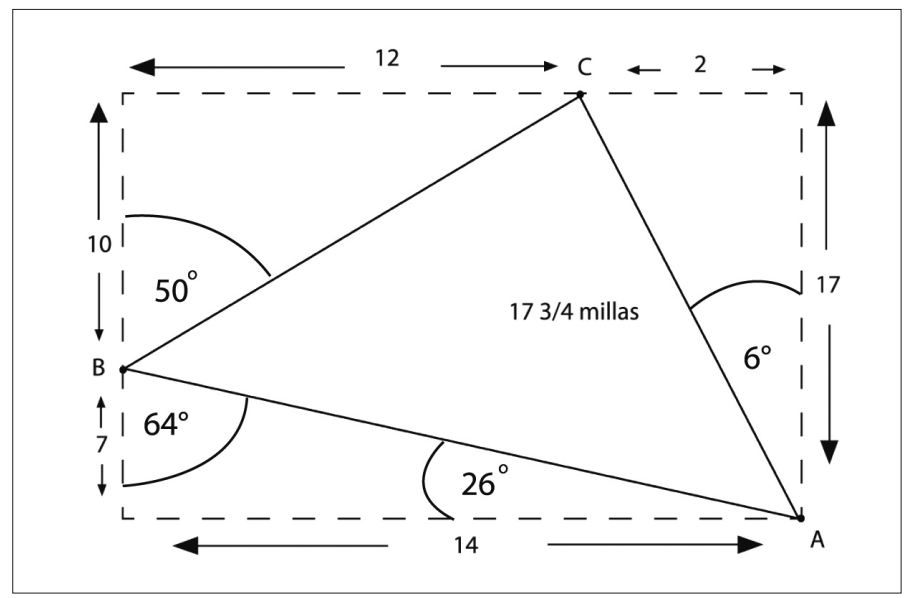

Fuente: Elaboración propia. 
Luego, estos ángulos permitirían calcular los dos ángulos interiores del triángulo ( $\mathrm{Fi}$ gura $N^{\circ} 6$ ). El ángulo del punto $A$, se puede obtener restando $90^{\circ}-6^{\circ}-26^{\circ}$ de lo que se obtiene como resultado $58^{\circ}$. Asimismo, el ángulo del punto $B$ se puede obtener restando $180^{\circ}-64^{\circ}-50^{\circ}$ de lo que se obtiene como resultado $66^{\circ}$.

Figura $\mathrm{N}^{\circ} 6$

Cálculo de los ángulos interiores

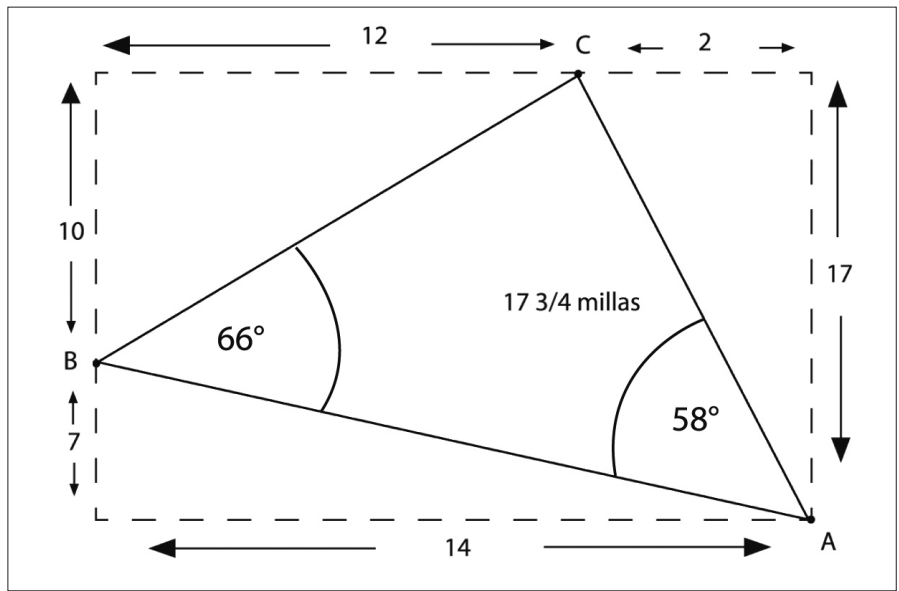

Fuente: Elaboración propia.

Calculados estos ángulos interiores se podía aplicar el teorema del seno, el cual establece la existencia de una relación proporcional entre los lados y ángulos de un triángulo. De esta manera, el seno de $66^{\circ}$ dividido por la medida del lado 17 3/4 millas debía ser igual al seno de $58^{\circ}$ dividido la medida del lado BC. El resultado de esta operación es que la medida del lado BC es igual a 15.7 millas.

La diferencia entre nuestro resultado y el obtenido por Moraleda, creemos que se debe a que, como indican los manuales de navegación de la época, los navegantes utilizaban tablas para resolver los problemas trigonométricos como los que se les presentaban, en este caso para deducir la arcotangente y el seno de ciertos ángulos, con lo cual las aproximaciones utilizadas en la época eran distintas a las que usamos en este caso para resolver el problema.

\section{Sondaje}

Además de la resolución de los cuatro términos básicos del pilotaje, Moraleda durante sus viajes entre el puerto de Guayaquil y Sonsonate realizó mediciones para conocer la profundidad del mar y la calidad del fondo. Para esto utilizó la sonda, que consistía en un cordel dividido en partes iguales por nudos que señalaban las brazas de profundidad. En el extremo esta tenía un escandallo con una parte cóncava que permitía conocer la calidad del fondo (Barreda, 1786: 151-153).

Para que fueran más precisos los resultados, las instrucciones ordenaban a Moraleda realizar el sondaje cuando el mar estuviera en calma.

Su diario de viajes nos permite establecer algunas características de las operaciones de sondaje. En primer lugar, el piloto solo daba a conocer los resultados de estas mediciones cuando se encontraba navegando cerca de la costa, cuando daban fondo durante la 
singladura o cuando estaban próximos a fondear en algún puerto. En estas situaciones, Moraleda detalla que las operaciones son realizadas constantemente a cortos intervalos de tiempo:

"A las 3 11/2 (...) seguimos con la sonda en la mano por fondos de 4 a 5 brazas ya arena, ya piedras gruesas, ya cascajo menudo; a las 3 3/4 habiendo sondado 4 brazas escasas fondo arena parda, viramos por avante en vuelta del Oeste, y a corto rato aumentamos el fondo a $4 \quad 1 / 2$ brazas y sucesivamente hasta 5 1/2 arena fina; a las $43 / 4$ hallándonos a poco más de $1 / 2$ milla de la costa de Lagartero, caímos de pronto en 4 brazas escasas" (Moraleda, día 23 a 24 de abril de 1804).

Las singladuras en las cuales el piloto no detalla los resultados del sondaje, normalmente cuando está en altamar, es posible encontrar alusiones al color de las aguas que le permitieron deducir la profundidad de estas:

"Amaneció el cielo toldado, los horizontes cargados, el viento flojo por el SE, y la mar Ilana y tan teñida que parecía de muy poco fondo" (Moraleda, día 27 a 28 de diciembre de 1802).

La práctica del sondaje también nos permite establecer que los resultados de estas mediciones junto a las que manejaba el piloto eran útiles para que pudiera deducir la ubicación de la nave:

"A la 1 de la mañana notando por el escandallo que íbamos, más bien atrasando que adelantando, y siendo poco viento del SSO que había contraído, dimos fondo en 6 $1 / 2$ brazas sobre lama" (Moraleda, día 12 a 13 de enero de 1804).

Creemos que esta técnica se volvió fundamental en la navegación de Moraleda por dos razones. En primer lugar, y como ya hemos señalado, su comisión estaba destinada a reconocer dos bajos, para lo cual además de situarlos astronómicamente, era necesario saber la profundidad y la calidad del fondo, con el objetivo de dar a conocer el porte de las embarcaciones que podían pasar por la zona y las precauciones que estas debían tener a su paso. Esto se relaciona estrechamente con el objetivo de su comisión que era precisamente mejorar la seguridad de la navegación de aquellas costas.

Las mediciones en el reconocimiento de los bajos eran realizadas a distintas horas, cuando la marea estaba alta, media o baja, de manera de poder identificar la profundidad según las características del mar en cada momento (v.g. Moraleda). Además, la importancia del sondaje queda reflejada en que Moraleda durante los reconocimientos organizó comisiones destinadas exclusivamente a sondar:

"Comisionamos al Teniente de Fragata don Juan de Pascual y Eraso, con el Piloto don Antonio Media-Aldea, para que con la lancha fuese reconociendo y notando los fondos, al rumbo del sur hasta tierra, y luego se dirigiesen costeándola al Oeste; al mismo tiempo ordenamos al Alférez de Fragata don Carlos Tiscar, y al Guardia Marina don José Morales, con el Pilotín, don Domingo Morales, para que practicase la misma diligencia al rumbo del Oeste, hasta la costa, y siguiesen luego orillándola hacia el SE" (Moraleda, día 22 a 23 de abril de 1803).

En segundo lugar, el sondaje era importante para el reconocimiento de las aguadas, pues conocer las características del agua resultaba fundamental para la provisión de esta durante los viajes. Por esto, Moraleda además de preocuparse de la calidad y profundidad del agua cuando realizaba los reconocimientos, prestaba atención a si el agua era salubre o no:

“El día 22 noticiosos por el Práctico Muñiz, que inmediato a nuestra aguada estaba el río de San Juan de Coyba (es el que tuvimos por pequeño estero salado, cuando levantamos este plano la campaña pasada), y que puede entrar el bote en él, aún a bajamar (...) comisioné al Teniente de Fragata don Juan de Pascual, lo ejecutase internándose cuanto pudiese, con el Piloto Media Aldea y el Práctico, lo que verificaron dirigiéndose al río (no se indica a la vista haberlo) con el pequeño bote (...) todo por agua salada hasta estar cercanos al punto en que no pudo ya nadar el bote, que la encontraron salobre por mezclada con la dulce del río (excelente)" (Moraleda, día 19 a 20 de marzo de 1804, acaecimientos en el puerto). 
De esta manera, la práctica científica de Moraleda no estaba guiada solo por el objetivo de identificar las características de los accidentes naturales, sino que también se basaba en la identificación de los elementos que permitían conservar la salud de los tripulantes.

\section{Demarcaciones y triangulaciones}

Moraleda utilizaba los métodos descritos para realizar las demarcaciones, es decir, fijar la situación de algunos puntos terrestres a partir de su rumbo, latitud y longitud. Esto se combinaba con operaciones geodésicas, que consistían en la formación de triángulos por medio de los cuales se unían los puntos demarcados. Luego, se calculaban las distancias entre ellos, lo que posteriormente permitía levantar una cartografía de los lugares.

El piloto durante su viaje a las colonias centroamericanas realizó dos tipos de demarcaciones, desde el mar hacia un punto en tierra firme $y$, en segundo lugar, una vez desembarcados y estando en la costa hacia otro punto en la tierra. Las primeras, eran realizadas por el navegante en forma diaria, salvo algunas excepciones en que llevó a cabo una navegación de altura y por lo tanto, no vio tierra. Este procedimiento era realizado por medio de la fijación de puntos notables, es decir, lugares que pudieran ser vistos desde otras tres estaciones, por lo cual se elegían cerros, islas, entre otros. Estos puntos notables constituirían los vértices del triángulo.

Las instrucciones ordenaban a Moraleda que lo primero que tenía que hacer a la hora de realizar las demarcaciones era acercarse al punto notable lo más posible, de manera de evitar errores de vista que influyeran en el resultado. El siguiente paso, era observar los horarios por medio del reloj y establecer la longitud del punto, que debía verificarse según el movimiento diario (Ugarte, 1802: capítulo 3, artículo 43).

Sin embargo, y a pesar de lo dispuesto por las instrucciones, lo primero que hizo el piloto al realizar las marcaciones fue determinar el ángulo del rumbo de los puntos con respecto a la nave. Esta medida no estuvo ausente en ninguna de las demarcaciones que ejecutó. Generalmente, determinaba la distancia del punto con respecto a su nave o a otro punto que hubiese demarcado. Si bien el piloto no hace mención del método que usó para obtener la latitud y longitud de dichos puntos, estas medidas se podían obtener a partir del rumbo y la distancia. Sin embargo, creemos que la longitud fue también obtenida por medio de la observación de las alturas del Sol, ya que las demarcaciones eran realizadas a los mismos puntos, una a las 6:00 hrs., luego a las 12:00 hrs. y finalmente a las 18:00 hrs. Al mediodía el margen de error en la obtención de la longitud era menor debido a que el Sol se encontraba más cerca del cenit. De esta manera, también se reducía la posibilidad de error en el cálculo de la distancia cuando esta no era medida por el piloto (Fidalgo, 1790: 164). Además, la práctica de realizar las mediciones a horas iguales con respecto al mediodía era la misma que utilizaba para conocer la longitud del lugar en el que se encontraba la nave, con lo cual creemos que este término era obtenido por observación más que por el uso de relojes, lo que también se puede relacionar con el hecho de que el reloj $\mathrm{N}^{\circ} 262$ no funcionaba, con lo cual no se podían unificar el método de las marcaciones entre la Castor y La Alavesa.

En la Figura $\mathrm{N}^{\circ} 7$, a partir de las demarcaciones realizadas en la singladura del 20 a 21 de abril de 1803, se muestra cómo realizaba el procedimiento. Al mediodía el Piloto señala que

"A las 6 se demarcó el morro de Puer$\cos$ al $\mathrm{N} 48^{\circ} \mathrm{O}$, y la punta de Mariato, tierra más Oeste a la vista, al $\mathrm{N} 81^{\circ} \mathrm{O}$, a distancia de la costa como $3 \quad \frac{1}{2}$ a 4 leguas (...) a las 8 aclaró algo y se vio la punta de Mariato al N $29^{\circ} \mathrm{O}$, a distancia de $4 \frac{11 / 2}{2}$ a 5 leguas (...) Al mediodía se demarcó el morro de Puercos al $\mathrm{N} 36^{\circ} \mathrm{E}$, punta de Mariato al $\mathrm{N} 12^{\circ} \mathrm{O}$, y la que me pareció punta de Montijo al $\mathrm{N} 27^{\circ} \mathrm{O}$, distancia de Mariato como 5 a 6 leguas (...) El rumbo directo de la singladura fue $\mathrm{S} 74^{\circ} 15^{\prime} \mathrm{O}$, distancia $366^{1 / 2}$ millas, no pudo observarse la latitud y fue la llegada de estima $6^{\circ} 56^{\prime} 30^{\prime \prime}$, y la longitud $74^{\circ} 16^{\prime} 40^{\prime \prime},(\ldots)$ por las últimas demarcaciones la latitud de llegada $6^{\circ} 56^{\prime} 30^{\prime \prime}$ y la longitud 74¹3'45" (Moraleda, s/f: día 20 a 21 de abril de 1803). 
Figura $N^{\circ} 7$

Proceso de demarcación desde mar a puntos notables en la tierra

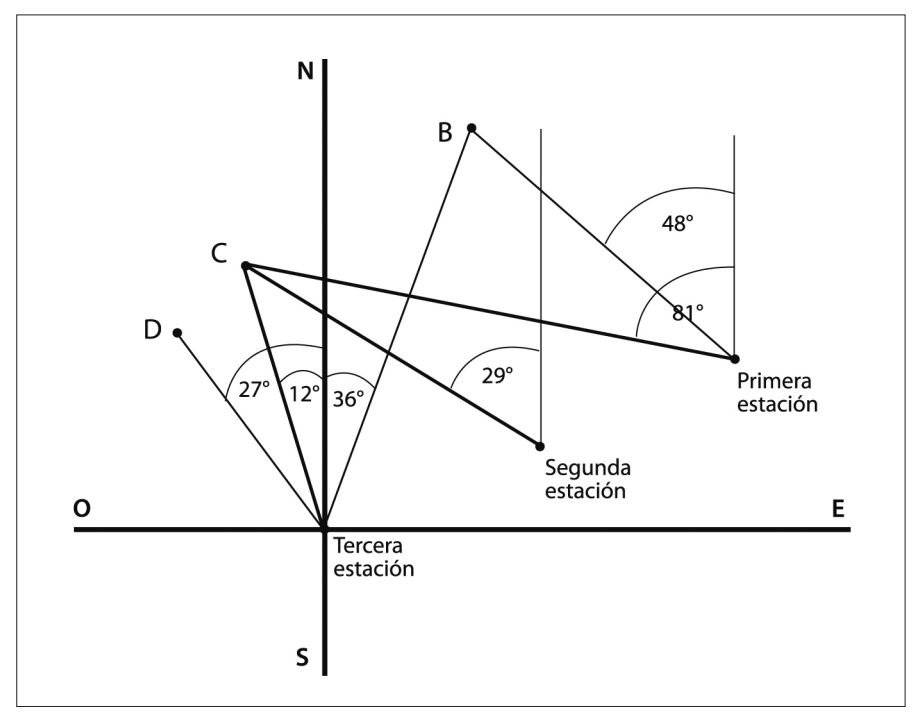

Fuente: Elaboración propia.

Las estaciones corresponden al punto desde donde mira el piloto, $\mathrm{B}$ al morro de Puercos, C a la punta de Mariato y D a la punta de Montijo. El punto que fue tomado como referencia en todas las estaciones es el C hacia el cual son calculadas las distancias desde la nave. En la tercera estación el piloto distinguió un nuevo punto que era la punta de Montijo, el cual agregó a los resultados obtenidos durante esa singladura. Junto a esto, el piloto indica que la latitud y longitud fueron calculadas por estima, de lo cual se deduce que para cada estación tenía el resultado aproximado de la situación de la nave. Por las últimas demarcaciones, obtuvo al mediodía la longitud y la latitud del punto en el que se encontraba. Las diferencias entre estas coordenadas a su vez le permitían conocer la distancia recorrida durante esa singladura.

Al tener estas coordenadas, los ángulos y las distancias desde la nave a $\mathrm{C}$, pudo determinar la medida de las distancias entre el punto $C$ y el $D$, como también desde el punto $\mathrm{C}$ a B. El método consistía en la formación de triángulos rectángulos para poder aplicar los principios de la trigonometría, y en los casos en que no podía formar triángulos rectángulos, pero sí contaba con la medida de dos ángulos y un lado podía aplicar por ejemplo, el teorema del seno que, como ya se ha mencionado, consistía en una proporción entre el seno de la medida de un ángulo y la longitud de su lado opuesto.

La resolución de este triángulo permitía resolver otros que se formaban continuando las demarcaciones a puntos notables y enfilándolas con respecto al primer triángulo. Por lo tanto, la resolución de la primera figura era un factor fundamental para lograr la exactitud en los resultados.

Si a estos datos se le agregaba los resultados de las mediciones de latitud y longitud por medio de los métodos señalados anteriormente, los distintos puntos podían quedar situados para luego ser cartografiados.

En algunas ocasiones el piloto no pudo practicar su método a las horas que acostumbraba. Cuando esto ocurría Moraleda daba a conocer la nueva hora en que realizaba las marcaciones y la causa por la cual las llevaba a cabo en ese momento:

"A las 5, por irse oscureciendo los objetos demarcables, se hicieron las siguientes mar- 
caciones, la medianía de la isla Santa Clara al $\mathrm{N} 26^{\circ} \mathrm{E}$, el cerro de las Animas al $\mathrm{N} 39^{\circ} \mathrm{E}$ y la punta de Malpaso al S $8^{\circ} \mathrm{O}^{\prime \prime}$ (Moraleda, día 9 a 10 de febrero de 1803).

En situaciones como las descritas, el piloto debía efectuar el mismo cálculo descrito anteriormente y determinar la situación de su nave con respecto a los objetos demarcados. A pesar de que había realizado el proceso una hora antes de lo previsto, continuó su patrón de conducta y las siguientes marcaciones fueron realizadas a las 6:00 hrs. y al mediodía.

El segundo tipo de demarcación que llevó a cabo Moraleda fueron las que realizó fuera del barco. Para explicarlas e identificar un patrón de conducta tomaremos como ejemplo su estadía en la ensenada de Damas.

Lo primero que hizo la tripulación cuando desembarcó fue realizar un reconocimiento del terreno de manera de poder identificar sus características:

"Pasamos a tierra todos los oficiales, a reconocer el terreno, para elegir, el que fuese más a propósito a las operaciones precisas, para levantar el plano de este puerto, que es el que más se preconiza, y único de estas costas que nos es conocido por noticias"13.

Esta elección resultaba fundamental para lograr la exactitud de los resultados, pues el lugar escogido debía proveer las condiciones necesarias para poder calcular la longitud y la latitud sin problemas y asegurar el funcionamiento de los instrumentos. Asimismo, era importante que el terreno tuviera una elevación adecuada para no influir en los cálculos de arrumbamientos, permitiera la provisión de agua y fuera cercano a la costa para realizar enfilaciones de los puntos de esta, entre otros.

Un día después de su llegada encuentra un lugar que cumple las condiciones:

"El 26 habiendo advertido que la pleamar dejaba una corta playa de arena, con monte

13 Acaecimientos de nota ocurridos durante la estancia en la ensenada de Damas. muy bajo interior al SE de nuestro surgidero, cerca de la punta que Ilamamos del Puerto, pasamos a reconocerla, y en efecto lo elegimos para situar las tiendas, a lo que inmediatamente, procedimos armándolas en lugar donde ni aun el flujo de las mareas de sisigias indicaba llegar" (Moraleda).

Luego de instalarse, la tripulación dio inicio a las operaciones trigonométricas y astronómicas. El primer paso era medir una base para la resolución del primer triángulo. Una vez formado este, del cual el piloto dio a conocer dos de sus puntos notables, la punta del Puerto y la del Corrillo, se calculaban las distancias entre un lado y otro por medio de operaciones trigonométricas. A pesar de haber obtenido las distancias por dicho método, el piloto las corroboró por medio del uso de sus instrumentos, lo que muestra su afán por la exactitud:

"Resuelto el primer triángulo de la base medida, cuyo lado mayor dio las distancias entre la punta del Puerto y la del Corillo, y es boca del estero de las tiendas, resolvimos rectificarla con la cadena mensoria, porque la baja-mar de sisigia lo proporcionó y al efecto nos dirigimos oficiales y pilotos, y verificada la medida material de dicho lado, solo se halló diferir 9 varas de la trigonométrica, cantidad bien despreciable a la verdad, para el plano de un puerto".

Resuelto este primer triángulo y corroborado los resultados, continuaron realizando enfilaciones para formar una cadena de triángulos que, como se ha explicado, se resolvían en base a los resultados obtenidos a partir del primero.

Paralelo a esto se organizaban comisiones que debían realizar las demarcaciones desde la costa, de manera de obtener la mayor cantidad de enfilaciones posibles desde distintas estaciones, lo que permitía aumentar la exactitud de los resultados.

Además, tal y como lo señalaban las instrucciones, los reconocimientos incluyeron sondajes, descripción de vientos y de corrientes, movimientos de las mareas, entre otros. Particularmente en esta zona llama la atención el énfasis que pusieron en el estudio de las aguadas, sus orígenes, ubicación y calidad 
del agua, lo que se explica por la abundancia de estas en los lugares cercanos al puerto y el afán del piloto por encontrar una aguada en este que facilitara e hiciera más cómodo el trabajo de abastecimiento de las naves.

Por último, Moraleda dio a conocer la forma en que obtuvieron la latitud y longitud exacta del puerto. El método utilizado para la obtención de la latitud fue el cálculo diario a bordo de la nave, luego a partir de todos los resultados se estableció una latitud media de $7^{\circ} 23^{\prime} 55^{\prime \prime}$, resultado que luego se rectificó en tierra por medio del uso del reloj $n^{\circ} 154$ de Arnold en el mismo lugar donde se observaba la longitud, obteniendo como resultado final $7^{\circ} 23^{\prime} 20^{\prime \prime}$. Como se ve, la diferencia entre el resultado alcanzado por medio de sus observaciones y el obtenido por el reloj es de treinta y cinco segundos, lo que muestra la exactitud con que el piloto llevaba a cabo sus cálculos. La longitud final del surgidero quedó establecida por medio del uso del reloj en $75^{\circ} 9^{\prime} 51^{\prime \prime}$. Este resultado a su vez era necesario enfilarlo con respecto a la longitud de base que se había tomado en la isla Gícara Grande para lo cual se realizaban operaciones trigonométricas que dieron por resultado para dicho surgidero la longitud de $75^{\circ}$.

\section{Las cartas}

Durante su comisión en Centroamérica, Moraleda utilizó cartas y planos que le permitieron ubicarse y que fueron la base de información sobre la cual realizó los reconocimientos. Al comienzo de su diario de viaje, el piloto señala que utilizaría las cartas publicadas por el Depósito Hidrográfico el año 1800, entre las cuales se encontraban las elaboradas por Alejandro Malaspina durante su expedición entre los años 1789 y 1794, en las naves Descubierta y Atrevida (Moraleda, advertencias 7). Además de ser un apoyo para el desenvolvimiento de la comisión, las instrucciones ordenaban a Moraleda rectificar

"las posiciones de varios puntos (...) se esmerará ahora en averiguar las determinaciones geográficas de los demás que intermedian, para formar al final, el exacto padrón y carta esférica de estas costas" (Ugarte, 1802: capítulo 1, artículo 6).

De lo anterior se desprende que Moraleda debía construir un conocimiento geográfico a partir de métodos científicos y de resultados de viajes anteriores, los cuales debían ser corregidos y completados. De esta manera, el piloto en su trayecto va corrigiendo la información que se tenía de ciertos lugares, como la isla de Lobos al norte del Perú, de modo tal que la comparación con el trabajo de la expedición de Malaspina se transformó en parte esencial de su quehacer científico.

Las diferencias más amplias entre los resultados de Moraleda corresponden a las obtenciones de longitud en la navegación de la costa comprendida entre el cabo Corrientes y Panamá. El piloto señala que las observaciones por medio del uso del reloj "han resultado unas $10^{\prime}$, y otras $11^{\prime}$ equinocciales al Occidente de las que señalan las cartas; pero en estas está situada aquella por operaciones o resultados trigonométricos, y no es aún tiempo de decidir de la bondad de dicha recomendada máquina" (Moraleda, sábado 4 a 5 de marzo de 1803). La diferencia a la que hace alusión Moraleda actualmente corresponde a 21 kilómetros aproximadamente y se puede explicar por las dificultades que experimentó Malaspina en su trayecto por la zona. El método del italiano consistía en basarse en la longitud obtenida en Guayaquil y seguir enfilando los puntos hacía el norte con respecto a ese resultado. Sin embargo, durante su navegación el tiempo se presentó nublado y lluvioso, lo que dificultó las observaciones al Sol para deducir la latitud y la longitud $y$, por otra parte, las corrientes lo desviaron constantemente de su rumbo. Además, los relojes entre ambas corbetas tenían una marcha desigual y las diferencias entre las longitudes obtenidas por estima y por observación a puntos como el cabo Corrientes habían sido de treinta minutos.

Las condiciones de navegación descritas impidieron que Malaspina pudiera realizar una navegación más cercana a la costa, y mientras su cercanía a los puntos notables que demarcó, como los Altos de San Francisco, era de leguas, la distancia que señala Moraleda era de millas, con lo cual el piloto español pudo precisar mejor la ubicación de ciertos accidentes. La distancia con la que el navegante italiano realizó las demarcaciones también influyó en la representación de la dirección de las costas entre San Francisco de Solano y el cerro del Sapo, que según lo 
señalado por el piloto español "hacen más ensenada al Este, que la que manifiestan las cartas del depósito; de forma que por los puertos del morro Quemado o Túpica, y el de Piñas que tenemos al Este se avanza de 4 a 5 milla más al oriente" (Moraleda, s/f: día 8 a 9 de marzo de 1803). A continuación el piloto señala que la distancia de 10 a 12 leguas con la que navegó Malaspina impidió "detallar la costa con la precisión necesaria" (Moraleda, s/f: día 8 a 9 de marzo de 1803).

Quizás el mayor error que corrigió Moraleda a Malaspina ocurrió en su salida desde el puerto de Damas para el de Guayaquil cuando bordearon la isla Gorgona, lo que también se relaciona con el hecho de la posibilidad que tuvo el español de realizar una navegación más cercana a la costa (Figura $\mathrm{N}^{\circ}$ 8). Para realizar la corrección de la situación de la isla, el piloto no solo consideró los resultados del viaje que estaba llevando a cabo, sino que también se basó en sus experiencias anteriores en la zona. A partir de esto, estableció dos tipos de errores en cuanto a la información que se manejaba de este lugar. En primer lugar, respecto a su situación geográfica:
"Por nuestras observaciones de latitud repetidas el año de 1800 en su fondeadero, y fuera de él a pequeñas distancias al Norte y Oeste de ella, y por las hechas hoy a su vista por cuatro observadores, que sólo ha diferido 1'30" entre el más u el menos, está en latitud $2^{\circ} 57^{\prime} 00^{\prime \prime}$ Norte, $4^{\prime}$ más de lo que la señalan nuestras cartas pues la sitúan en $2^{\circ} 52^{\prime} 30^{\prime \prime}$ su medio" (Moraleda, día 21 a 22 de mayo de 1803).

Además de fijar la situación del territorio por medio de la práctica científica, el piloto incluyó una representación de la isla y una descripción de esta que había realizado durante su estadía anterior. Al hacer esto, Moraleda fue actualizando la información que se había generado años antes, lo que además de mostrarnos la confianza que el piloto tenía en sus propios resultados, muestra cómo se va desarrollando el conocimiento más profundo de un lugar, que no solo considera la información existente, sino que también las prácticas previas del mismo piloto, las cuales influyeron en la forma en que realizó las mediciones y en los resultados obtenidos.

Figura $\mathrm{N}^{\circ} 8$

Bosquejo horizontal y vistas de la Isla Gorgona cercana a las costas de Popayan en el mar Pacífico. Formado en 1800 por el teniente de fragata de la Real Armada don José de Moraleda, y situada su medianía en latitud N 257'30" por repetidas observaciones hechas en los años 1800, 1803 y 1804.

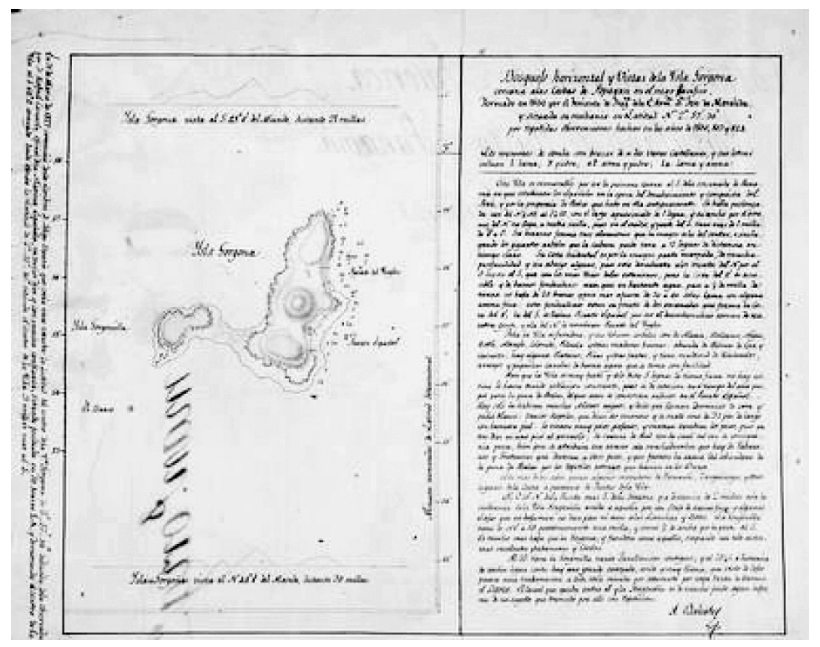

Fuente: Museo Naval de Madrid. 
El segundo aspecto en el cual el español corrigió a Malaspina, se relaciona más con una visión mítica del navegante italiano acerca del lugar que recorría, a su juicio la Gorgona:

"Merece la mayor atención entre los que navegan desde Acapulco, o Panamá, a Paita (...) al recalar, o no a el S. de el, decide la felicidad del viaje; y liberta a el Navegante de Engorgonarse, o ser arrastrado por las Corrientes" (Malaspina, 1987: 208).

Frente a esta concepción de la isla, el italiano no hizo un reconocimiento más profundo de esta, no se acercó y menos fondeó para examinarla cabalmente, a diferencia de lo que hizo Moraleda, quien por medio de su práctica científica le dio una justificación teórica a las condiciones geográficas que existían en la isla y que daban origen al mito del que de ella se tenía:

"La palabra "engorgonarse", (...) no la usarán seguramente los navegantes sensatos en un sentido tal, por lo respectivo a la náutica; pues la Gorgona como todos los lugares del globo que habitamos, tiene sus vientos periódicos constantes, y regulares variaciones de que se aprovechan oportunamente los inteligentes, absolviendo la distancia dicha en doce o quince días, y dan al más solemne desprecio semejante groseras opiniones, que oprobian hasta la razón natural" (Moraleda, día 21 a 22 de mayo de 1803, nota).

Lo anterior, no solo nos muestra que Moraleda corrigió creencias asentadas en los navegantes a partir del método científico, sino que también aportó una solución para contrarrestar los problemas que originaban las características geográficas del lugar.

\section{Conclusiones}

El análisis de la práctica científica del piloto español por el Pacífico centroamericano nos permite establecer ciertas características de su método. En primer lugar, es necesario recalcar lo sistemático que era el piloto en la práctica de la navegación científica, pues las observaciones y mediciones no solo eran realizadas en forma diaria, sino que varias veces durante el día y a horas determinadas. La determinación de los horarios también refleja la uniformidad del método, pues se esperaba a que el astro, generalmente el Sol, estuviera siempre a la misma altura para poder realizar las mediciones. De esta manera, los distintos resultados fueron obtenidos a partir del paso del astro por un mismo meridiano, regla respecto de la cual hubo pocas excepciones.

Los métodos utilizados por Moraleda permiten ver la exactitud con la que el piloto intentaba realizar su labor. Es lo que sucede, por ejemplo, con el cálculo de la distancia, pues si bien la comisión contaba con relojes para medir la longitud, los cuales permitían deducir la distancia por medio de la diferencia de meridianos, se siguió utilizando el método tradicional de la corredera. Asimismo, en muchas singladuras el piloto detalla que además de obtener los resultados por medio del uso de los instrumentos, realizaba sus propios cálculos matemáticos. Esto resulta importante si recordamos que el conocimiento científico desde el siglo XVIII estaba fundado en el uso de los instrumentos, pues estos tenían la facultad de poder ser trasladados de su contexto de origen, permitiendo su utilización en otros lugares, y por lo tanto, la posibilidad de ser replicados. También, los instrumentos científicos al establecer una uniformidad en el método, permitían la estandarización de los resultados (Bourguet, 2002: 1-19). La reiteración de las mediciones, además de vincular métodos matemáticos, con operaciones físicas y geodésicas, refleja la persistencia del piloto por la obtención de resultados más precisos, pues entre menos diferencia existiese entre los distintos cálculos menor sería el margen de error.

Estas características son las que permitieron a Moraleda transformarse en un referente para importantes viajeros, como Alexander von Humboldt. El prusiano utilizó la información obtenida por medio de su experiencia junto a Moraleda en la Castor para la elaboración de su obra, sobre todo para la descripción de la costa que va desde Lima hasta Guayaquil, y para la elaboración de una cartografía correspondiente a dicha zona. Por otra parte, utilizó los resultados de los viajes anteriores de José Moraleda, como el realizado a Chiloé, cuyo diario de viaje Humboldt calificó de "manuscrito precioso" y que le permitió dar a conocer las condiciones 
de ciertas rutas y la forma en que se debían realizar algunas navegaciones (Humboldt, 2002: 487). Si se recuerda el importante valor que Humboldt asignaba al método científico, entendido como planteamiento de una hipótesis, experimentación y a partir de esto el establecimiento de conclusiones o leyes generales, Ilama la atención que en su obra incluya información que él mismo no obtuvo empíricamente, lo que nos da cuenta de lo confiable que resultaban los trabajos de Moraleda para la época (Ramírez, 2000: 89110).

Por otra parte, la práctica científica del piloto refleja el carácter acumulativo del conocimiento geográfico de la realidad americana. Al basarse en las cartas elaboradas por Malaspina, Moraleda no solo fue corrigiendo y perfeccionando los conocimientos que se manejaban en la época, sino que también lo fue articulando y especificando por medio de la fijación de puntos intermedios. De esta manera, en la generación del conocimiento científico existió una dependencia y movilidad de la información disponible, creándose redes de información que fueron fundamentales para lograr la precisión del conocimiento.

Además, creemos que es necesario recalcar que si bien nos hemos referido casi únicamente a los métodos ejercidos por el piloto, junto a él observaban y medían un conjunto de personas que fueron indispensables en el quehacer del navegante. La presencia y las actividades Ilevadas a cabo en la Alavesa eran imprescindibles para la nave la Castor, tanto para realizar comparaciones como para uniformar los resultados. Además, como se ha explicado a lo largo del trabajo, en algunos métodos se hacía indispensable la presencia de otros observadores, lo que ayudaba a lograr una mayor exactitud en los resultados, evitando que el piloto tuviera que realizar por sí solo todas las mediciones. De aquí la importancia de las comisiones organizadas por Moraleda, que además de significar un ahorro de tiempo permitía un reconocimiento más profundo de las distintas zonas.

Lo anterior nos permite comprender la actividad científica y la generación de conocimiento como una actividad colectiva, en la que tras la individualidad del piloto que toma el papel de protagonista, trabajaba un grupo más amplio, que abarcaba la experiencia de los navegantes que le habían antecedido y la participación de las personas que formaban parte de la tripulación.

Además, queremos recalcar que la práctica científica de Moraleda en el Pacífico centroamericano responde a una determinada forma de entender y de realizar la ciencia. En un contexto en el que la corona española financiaba la navegación científica en las costas americanas para lograr el conocimiento geográfico, que era disputado y en torno al cual se producía rivalidad internacional, era necesario realizar los métodos con rigurosidad, de manera de lograr resultados lo más exactos posibles, que beneficiaran a la metrópolis. Es la ciencia al servicio del poder y el piloto actuando como funcionario del Estado, experimentando y reconociendo a partir de métodos científicos la costa centroamericana. De esta manera, creemos que la relación entre la metrópolis y las colonias, también se producía por medio del quehacer científico del piloto y los viajes que este realizó, lo que a su vez nos permite comprender la actividad científica como una actividad en movimiento (Ette, 2010: 321-338). Si bien los avances eran pensados en Europa, fueron en las coIonias americanas el lugar donde se hicieron los esfuerzos de experimentar y comprobar las innovaciones que se estaban produciendo en el Viejo Mundo.

Finalmente, el estudio de este caso refleja las posibilidades que ofrece la historia de la ciencia para abordar los viajes científicos no solo desde el punto de vista de sus resultados, sino también comprenderlos a partir de los elementos que lo conforman, en este caso, los métodos utilizados por el piloto. De este modo, este trabajo permite comprender la actividad científica desarrollada para el reconocimiento geográfico de América, como un quehacer cultural, vinculado al contexto y a las condiciones en las que se desenvolvieron las comisiones, rescatando la ciencia como un hecho social dispuesto a ser objeto de análisis histórico. 


\section{Referencias bibliográficas}

BARREDA, F. El marinero instruido en el arte de navegar especulativo y práctico. Segunda Impresión, 1786.

BOURGUET, M.N.; LICOPPE, CH. \& SIBUM, H. (Editors). Instruments, Trave and Science. Itineraries of precision from the seventeenth to the twentieth century. LondresNueva York: Routledge, 2002.

CISCAR, G. Cursos de estudios elementales de marina. Imprenta Real, Tomo IV, 1811.

DIRECCIÓN GENERAL DEL INSTITUTO GEOGRÁFICO NACIONAL. Instrumentos históricos del Instituto Geográfico Nacional. Madrid: Impreso en los talleres de la Dirección del Instituto Geográfico Nacional, 2002.

ETTE, O. El Cosmos de la vida: Alexander Von Humboldt y su obra mayor. En: SAGREDO, R. (Editor). Ciencia-Mundo. Orden republicano, arte y nación en América, Santiago de Chile: Editorial Universitaria, 2010.

FIDALGO, J. Lecciones de Geometría práctica dispuestas para la instrucción de los cavalleros del Departamento Guardias Marinas de Cádiz. Isla de León, 1790.

GUTIÉRREZ, M. "Expediciones en tiempos de Carlos IV. En: DIEZ, A.; MALLO, T; PACHECO, D y ALONSO, Á. (Coord.), La ciencia española en ultramar. Actas de la I Jornada sobre España y las expediciones científicas en América y Filipinas, Madrid, Doce Calles, 1991

MALASPINA, A. En: CEREZO, R. La Expedición Malaspina 1789-1794. Diario General del viaje. Madrid: Ministerio de Defensa- Museo Naval y Lunwerg, Tomo II, volumen $1^{\circ}$, 1987.

MORALEDA, J. Diario de viajes desde el Puerto del Callao a los de Guayaquil y Panamá, y de estos al reconocimiento y demarcación de las costas de Veragua, Rica, Nicaragua y Guatemala. Museo Naval de Madrid, 1802-1804
HUMBOLDT, A. Ensayo Político sobre el reino de la Nueva España. México D.C.: Porú́a, 2002.

MARTÍNEZ-CAÑAVATE, L. La Expedición Malaspina 1789-1794. Trabajos Astronómicos Geodésicos e Hidrográficos. Madrid: Lunwerg Editores, Tomo VI, 1990.

O'DONELL Y DUQUE DE LA ESTRADA, H. El viaje a Chiloé de José de Moraleda (1787-1790). Madrid: Editorial Naval, 1990.

PIMENTEL, J. La Física de la Monarquía: ciencia y política en el pensamiento colonial de Alejandro Malaspina (1754-1810). Aranjuez: Editorial Doce Calles, 1998.

PINTO, J.C. Historia General de Centroamérica. El Régimen Colonial (1524-1750). Madrid: FLACSO, 1993.

RAMÍREZ, S. El método científico en la obra americana de Alejandro de Humboldt. En: GUTIÉRREZ, A. (Coordinador). Ciencia, economía y política en Hispanoamérica Colonial. Sevilla: Consejo Superior de Investigaciones Científicas, 2000.

RIVERA, B. y MARTÍN-MERÁS, L. Cuatro siglos de cartografía en América. Madrid: Colecciones Mapfre, 1992.

SAGREDO, R. y GONZÁlEZ, I. La Expedición Malaspina en la frontera austral del Imperio español, Santiago de Chile: Editorial Universitaria, 2004.

SAGREDO, R. Navegación científica en el Mar del Sur. El piloto Moraleda (1772-1810). Revista de Historia Iberoamericana, 2008. Disponible en Internet: http:// revistahistoria.universia.cl/pdfs_revista/articulo_89_1250114582587.pdf

SELLÉS, M. Instituciones científicas ilustradas de la marina. En: DIEZ, A.; MALLO, T.; PACHECO, D. y ALONSO, Á. (Coordinadores). La ciencia española en ultramar. Actas de la I Jornada sobre España y las expediciones científicas en América y Filipinas. Madrid: Doce Calles, 1991. 
SELLÉS, M. Instrumentos de navegación. Del Mediterráneo al Pacífico. Barcelona: Lunwerg- Consejo Superior de Investigaciones Científicas, 1994.

SELLÉS, M. Navegación astronómica en la España del siglo XVIII. Madrid: Universidad Nacional de Educación a Distancia, 2000.

SOBEL, D. Longitud. La verdadera historia de un genio solitario que resolvió el mayor problema científico de su tiempo. Madrid: Editorial Debate, 1998.

UGARTE, T. Instrucciones que deberá observar el teniente de fragata José de Moraleda, con la corbeta Castor de su mando, y goleta Alavesa al del alférez de navío don Antonio Quartara, cuyos bajeles formarán la segunda división, que ha de emplearse en tareas hidrográficas desde el golfo de Panamá al puerto de Sonsonate, en cumplimiento de Real Orden de 1 de octubre de 1801. Museo Naval de Madrid, signatura MS 127.

VALDEVERDE, N. Actos de precisión. Instrumentos científicos, opinión pública y economía moral en la llustración española. Madrid: Consejo Superior de Investigaciones Científicas, 2007. 
